\title{
Subcritical patterns and dissipative solitons due to intracavity photonic crystals
}

\author{
Damià Gomila ${ }^{1,2}$ and Gian-Luca Oppo ${ }^{1}$ \\ ${ }^{1}$ SUPA and Department of Physics, University of Strathclyde, 107 Rottenrow, Glasgow, G4 ONG, Scotland, United Kingdom \\ ${ }^{2}$ Instituto de Física Interdisciplinar y Sistemas Complejos (IFISC,CSIC-UIB), Campus Universitat Illes Balears, \\ 07122 Palma de Mallorca, Spain
}

(Received 13 June 2007; revised manuscript received 21 September 2007; published 17 October 2007)

\begin{abstract}
Manipulation of the bifurcation structure of nonlinear optical systems via intracavity photonic crystals is demonstrated. In particular, subcritical regions between spatially periodic states are stabilized by modulations of the material's refractive index. An family of dissipative solitons within this bistability range due to the intracavity photonic crystal is identified and characterized in both one and two transverse dimensions. Nontrivial snaking of the modulated-cavity soliton solutions is also presented.

DOI: 10.1103/PhysRevA.76.043823

PACS number(s): 42.65.Tg, 42.65.Sf, 89.75.Kd, 05.65.+b
\end{abstract}

\section{INTRODUCTION}

The effect of background modulations in the nonlinear dynamics of broad-area photonic devices leads to control and stabilization of spatial structures $[1,2]$. Recently we have studied the formation of spatial structures in nonlinear optical devices with intracavity photonic crystals, i.e., a periodic modulation of the material's refractive index [3]. We observed that, for some values of the cavity detuning, the linear band gap of the photonic crystal inhibits modulational instabilities that lead to the formation of periodic spatial structures (patterns). This yields a gain of stability of the fundamental solution, resulting in straightforward potential applications for the control of spatial instabilities in broadarea photonic devices. In [3], however, we restricted ourselves to the case of supercritical bifurcations only. Here, we study the effects of a periodic modulation of the refractive index on the formation of subcritical structures. This case is especially relevant for the observation of dissipative (or cavity) solitons (CSs). CSs are miniature beams of light, selflocalized through the material nonlinearity and stored within an optical cavity [4]. They are robust features and can be used as elements for optical processing in semiconductorbased optoelectronics [5].

In regimes where CSs exist, we observe a very different behavior from that reported in [3], including localized structures in different subcritical regimes where the fundamental solution is stabilized by the intracavity photonic crystal. The periodic modulation, coupled to the nonlinear nature of our models, has powerful effects on the formation of subcritical patterns and allows for the existence of an additional family of CSs. This is an example of the nontrivial effects that a photonic crystal may have on nonlinear systems, beyond their usual linear effects on light propagation.

The paper is organized as follows. In Sec. II we introduce a useful model for a broad-area Kerr medium with an intracavity photonic crystal (ICPC) and briefly review supercritical bifurcations induced by the ICPC. In Sec. III subcritical bifurcations are found and characterized. These would not exist should the ICPC be removed from the device and are at the basis of the formation of "modulated" localized states or CSs. Details of the formation, shape, and stability of the modulated CSs are provided in Sec. IV, where we show that their presence can be better identified with Fourier filtering techniques. Analogies and differences between the modulated and the more traditionally shaped CSs are also discussed. Conclusions about the generality and universality of the ICPC-induced subcritical bifurcations and modulated CSs are presented in Sec. V.

\section{THE MODEL}

We consider an optical cavity containing a self-focusing Kerr medium and a linear medium with a spatially modulated refractive index [3]. This model is convenient for its simplicity but the phenomena discussed in the next sections extend to a variety of nonlinear optical cavities such as saturable absorbers, optical parametric oscillators, and lasers. In one transverse spatial dimension, the evolution of the slowly varying amplitude of the paraxial electric field $E$ is described, in the mean-field approximation, by [6]

$$
\partial_{t} E=-\{1+i[\theta+f(x)]\} E+i \partial_{x}^{2} E+E_{0}+i|E|^{2} E,
$$

where $\theta$ is the average detuning between the frequency of the pump and the frequency of the cavity, $f(x)$ accounts for the modulated refractive index in the transverse direction of the photonic crystal, $\nabla^{2}$ is the transverse Laplacian, and $E_{0}$ is the input field. Here we consider $f(x)$ to be a square function of amplitude $\alpha$ and wave number $k_{p c}$. In the absence of the photonic crystal $(\alpha=0)$ Eq. (1) has a homogeneous steadystate solution implicitly given by

$$
E_{s}=E_{0} /\left[1+i\left(\theta-I_{s}\right)\right],
$$

where $I_{s}=\left|E_{s}\right|^{2}$. Equation (2) has a single-valued solution for $\theta<\sqrt{3}$. Here we restrict ourselves to this range and use $I_{s}$ and $\theta$ as convenient control parameters. The homogeneous solution is stable for $I_{s}<1$ and becomes modulationally unstable at $I_{s}=1$ with critical wave number $k_{c}=\sqrt{2-\theta}$, leading to the formation of a stripe pattern.

For $\theta>41 / 30$, the transition associated with the modulational instability is subcritical $[6,7]$ so that stable homogeneous and patterned solutions coexist for intensities $I_{s}<1$, below the instability. Inside this subcritical regime, localized structures, the so-called cavity solitons, have been found. The existence and bifurcation diagrams of such structures have been studied in [8]. 


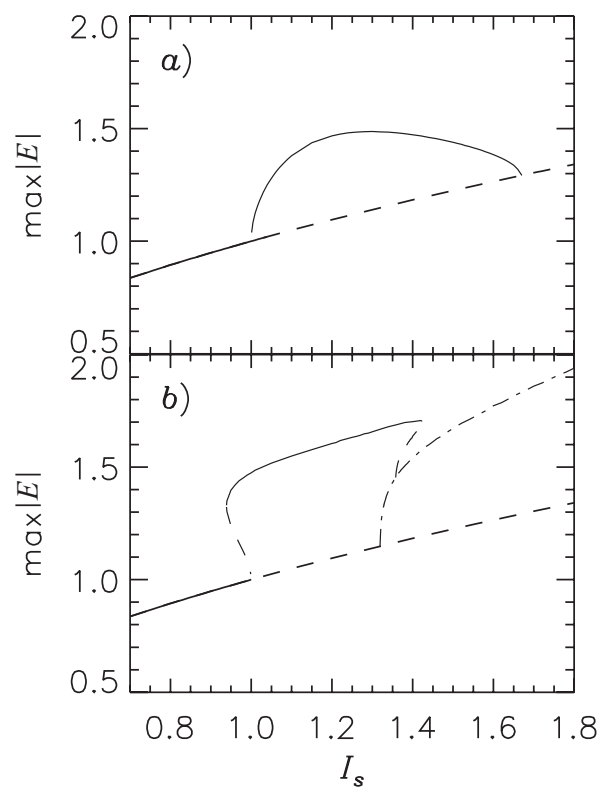

FIG. 1. Bifurcation diagram of a pattern with the critical wave number $k_{c}$ without ICPC $(\alpha=0)$. (a) Supercritical bifurcation for $\theta=-2$. (b) Subcritical case for $\theta=1.5$. Solid (dashed) lines indicate stable (unstable) solutions. The dot-dashed line in (b) shows the bifurcation line of a pattern with $k=2 k_{c}$, which is also unstable.

Figure 1 shows the bifurcation structure of the critical pattern for two values of the detuning, one below 41/30 and one above. In Fig. 1(b), for instance, the pattern coexists with the homogeneous solution for $0.939<I_{s}<1$.0. For later comparison, single-peak localized solutions exist for $0.9628<I_{s}<0.9715$.

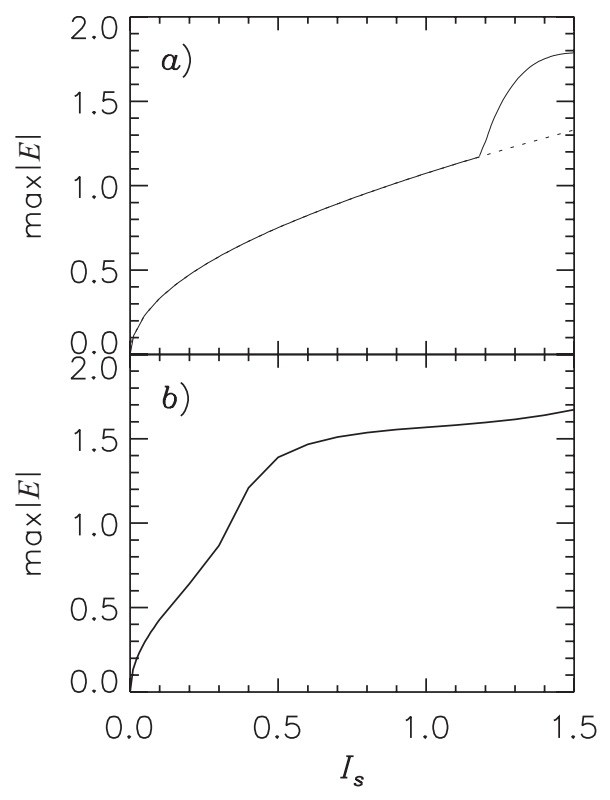

FIG. 2. Bifurcation diagram of the fundamental solution with ICPC for $\alpha=0.75$. $\theta=(a)-2.0$ and (b) 1.5 .

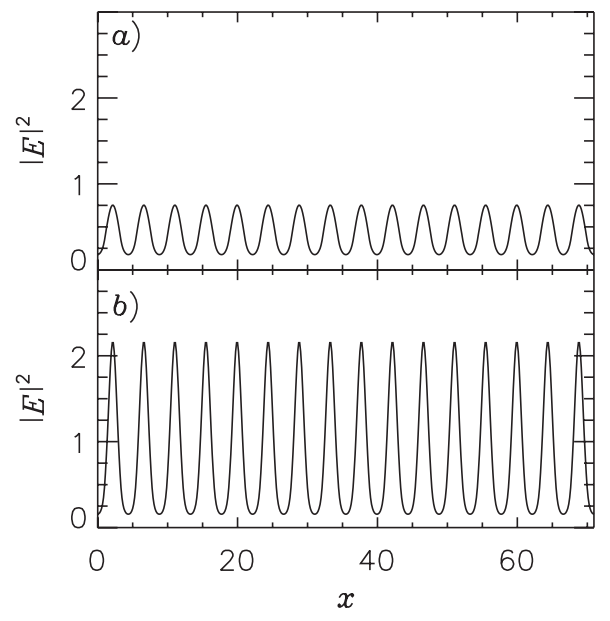

FIG. 3. Fundamental solution of the ICPC system with $\theta=1.5$ and $\alpha=0.75 . I_{s}=$ (a) 0.3 and (b) 0.6 .

\section{SUBCRITICAL PATTERNS}

In [3] we analyzed the effects of an ICPC on the formation of supercritical patterns. We found that periodic modulations with a large enough amplitude inhibited patternforming instabilities. When the ICPC is included in the simulations with a relatively high amplitude $[\alpha=O(1)]$ we observe the following scenario in the detuning range $-2<\theta<1.5$ (see Fig. 2).

(1) For negative or small positive values of $\theta$, the ICPC shifts the first instability of the homogeneous solution, and also the range of parameters where modulated solutions ex-
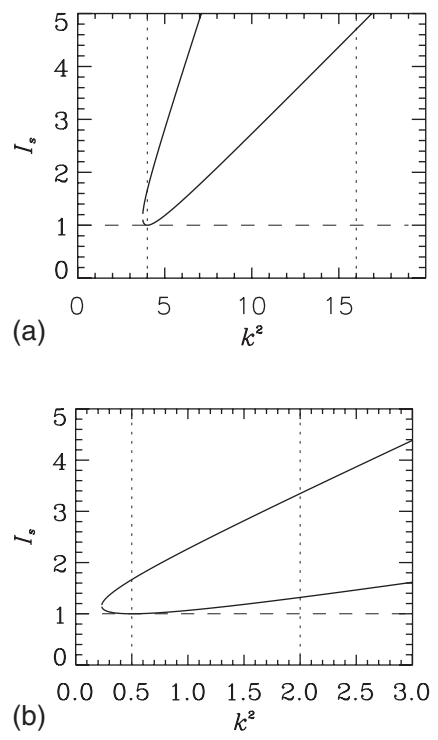

FIG. 4. Marginal stability curve of the homogeneous solution without photonic crystal $(\alpha=0)$ for $\theta=(a)-2$ and (b) 1.5. The horizontal dashed line shows the threshold for pattern formation. The two vertical dotted lines indicate the critical $k_{c}$ and photonic crystal $k_{p c}$ wave numbers. The vertical distance from the marginal stability curve to the threshold line is an indication of the stability of perturbations corresponding to that wave number. 


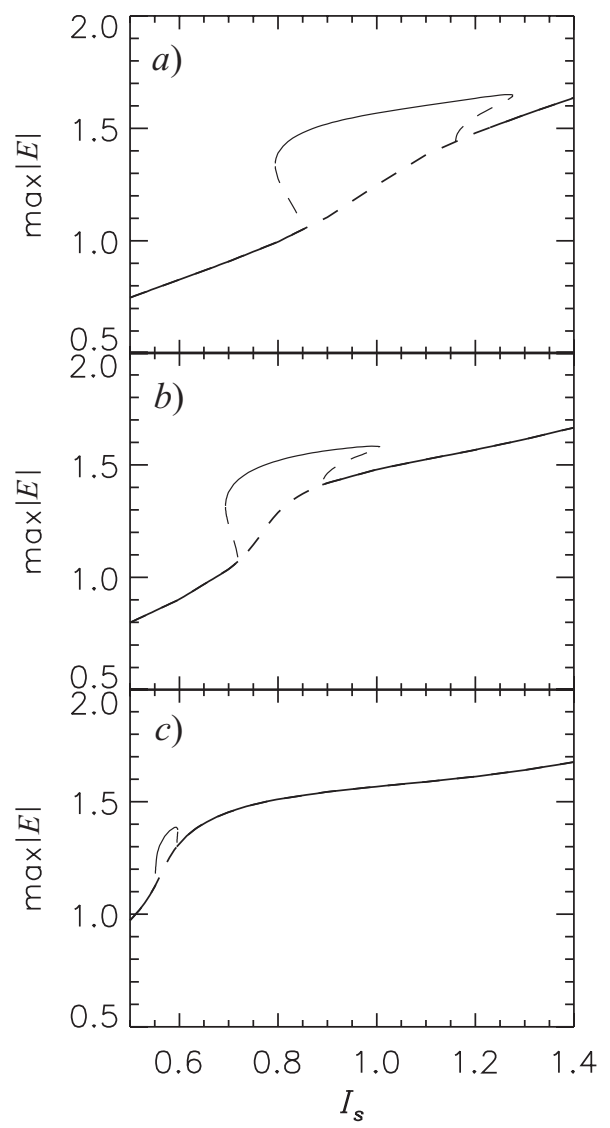

FIG. 5. Bifurcation structure of a pattern with the critical wave number in the presence of photonic crystals of amplitudes $\alpha=(\mathrm{a})$ 0.1 , (b) 0.2 , and (c) $0.4(\theta=1.5)$.

ist, to higher values of the input intensity without, however, changing the supercritical nature of the bifurcation [see Figs. 1(a) and 2(a)].

(2) For positive values of $\theta$ above 41/30, where one finds a subcritical instability without the ICPC [see Fig. 1(b)], the ICPC completely alters the bifurcation diagram and suppresses any instability and pattern solution [see the curve in Fig. 2(b) corresponding to $\alpha=0.75$ and $\theta=1.5]$. For these values of the parameters, one would in principle expect subcritical spatial instabilities, but the fundamental solution remains stable, although it abruptly modifies its profile. For $\theta=1.5$ [Fig. 2(b)], for instance, it changes from a lowamplitude modulation for low values of $I_{s}$ to a highly anharmonic pattern with twice the amplitude upon a relatively small increment of the intracavity intensity (Fig. 3). We note, again, that there is no coexistence between the fundamental solution and high-amplitude patterns.

We interpret this phenomenon as a resonance of the wave number $k=2 k_{c}$ with the modulation introduced by the photonic crystal $\left(k_{p c}=2 k_{c}\right)$. In [3] we studied the effects of a periodic modulation of wave number $k_{p c}=4$ on the formation of patterns in the detuning range $-6<\theta<1.73$. Pattern inhibition occurred when $k_{c} \sim k_{p c} / 2=2$, i.e., for values of the detuning around $\theta=-2$. In that case, the response of the system to the ICPC was very small, according to the large and negative decay rate of perturbations at $k=k_{p c}$ [see Fig. 4(a)]. The

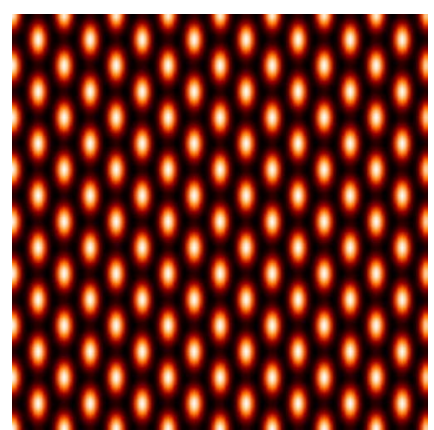

(a)

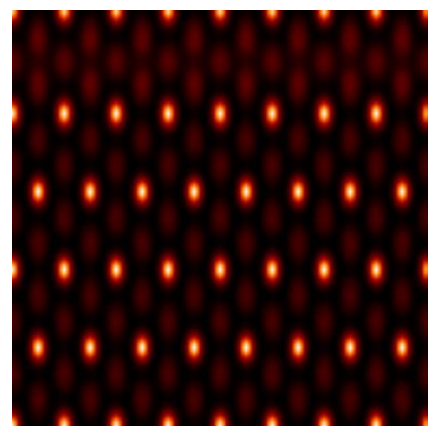

(b)

FIG. 6. (Color online) Near field of coexisting fundamental (left) and pattern (right) solutions in 2D for $I_{s}=0.7$ and $\theta=1.2$. Simulations have been performed on a $128 \times 128$ rectangular grid with $d k_{x}=0.097$ and $d k_{y}=\sqrt{3} d k_{x}$.

ICPC, however, had the property of modifying the dispersion relation of the cavity, introducing a band gap. Here, instead, we are interested in regimes where CSs exist. In our system, this takes place above $\theta=41 / 30$, and this corresponds to a situation where the critical wave number is small and the wave number $k_{p c}=2 k_{c}$ is only weakly dumped [see Fig. 4(b)]. In this case the mechanism for the band-gap inhibition of spatial instabilities (see [3]) fails and the system presents a strong response to the perturbation introduced by the modulation. A strong response to the ICPC enhances the amplitude of the fundamental solution, and destroys the formation of subcritical patterns at $k_{c}$.

For $\theta>41 / 30$ the ICPC progressively removes subcritical patterns for increasing values of the modulation amplitude $\alpha$. Figure 5 shows such transition for $\theta=1.5$ and values of $\alpha$ between those of Figs. 1(b) and 2(b), i.e., $\alpha=0.1,0.2$, and 0.4 .

When increasing the amplitude of the modulation $\alpha$ we observe a progressive stabilization of the fundamental solutions for large values of $I_{s}$ [Fig. 5(a)]. This mechanism is different from the band-gap inhibition studied in [3]. Here, the fundamental solution becomes stable through a locking with the periodic forcing introduced by the photonic crystal, similar to the case studied in [1]. In this case, however, the modulation is in the refractive index instead of in the input pump. Eventually, the fundamental solution becomes stable for all values of the pump except for values between the two bifurcation points that lead to the pattern with $k=k_{c}$ [Fig. $5(\mathrm{~b})]$. Both bifurcations are subcritical and the fundamental 


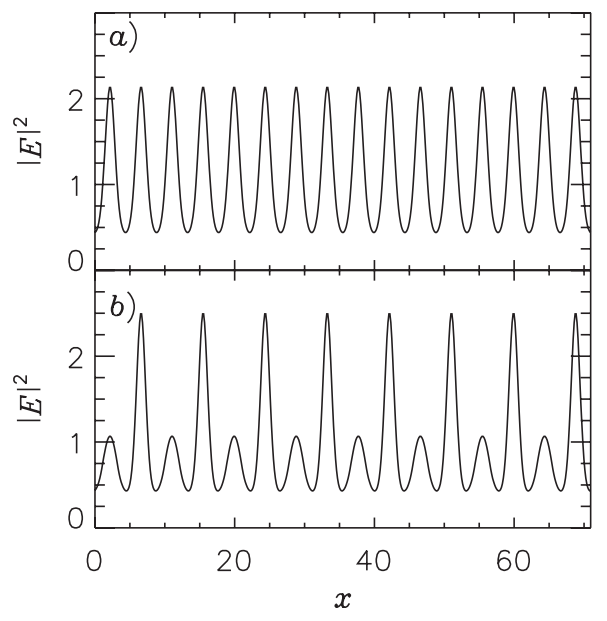

FIG. 7. Intensity profile of the coexisting (a) fundamental and (b) pattern solutions for $I_{s}=0.96$ and $\theta=1.5$ in the presence of a periodic modulation of amplitude $\alpha=0.2$.

solution stably coexists with the pattern in two different regions of the parameter space. In the absence of ICPC, the fundamental solution is typically (except for systems with peculiar symmetries) unstable in the subcritical region at large values of the input pump [Fig. 1(b)]. Remarkably, the new region of coexistence between the fundamental and the pattern solutions close to the reverse (second) subcritical bifurcation is much larger in parameter space than the first one. For instance, in the system without a photonic crystal, for $\theta=1.5$ the pattern and homogeneous solution coexist and are stable for $0.938<I_{s}<1.0$ while with a gentle periodic modulation (such as with $\alpha=0.2$ ) this region shifts to $0.6936<I_{s}$ $<0.72$ and a completely new and broader region appears for $0.891<I_{s}<1.006$. We note that the size of the second subcritical region is 0.115 as compared with 0.062 in the case without a photonic crystal. This is roughly an $85 \%$ increment, greatly enhancing the possibility of realizing localized

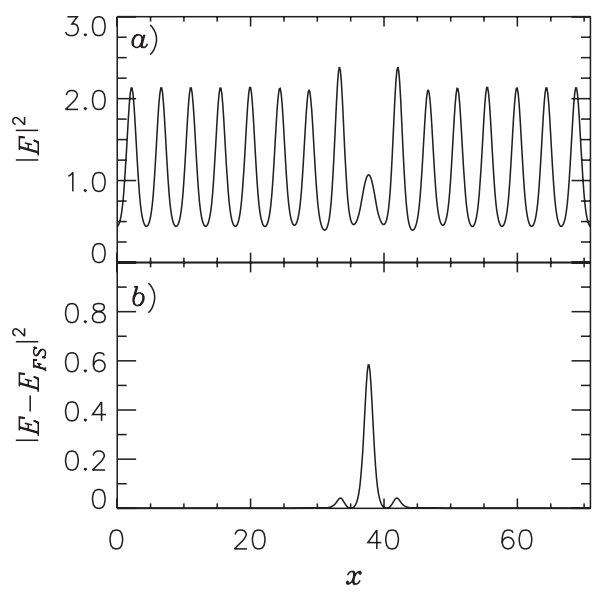

FIG. 8. (a) Intensity of a single-peak localized structure (CS) and (b) the intensity difference between the CS and the fundamental solution in the subcritical regime for large values of $I_{s}$. (b) Field difference (destructive interference) between the CS and the fundamental solution. Here $I_{s}=0.96, \theta=1.5$, and $\alpha=0.2$.
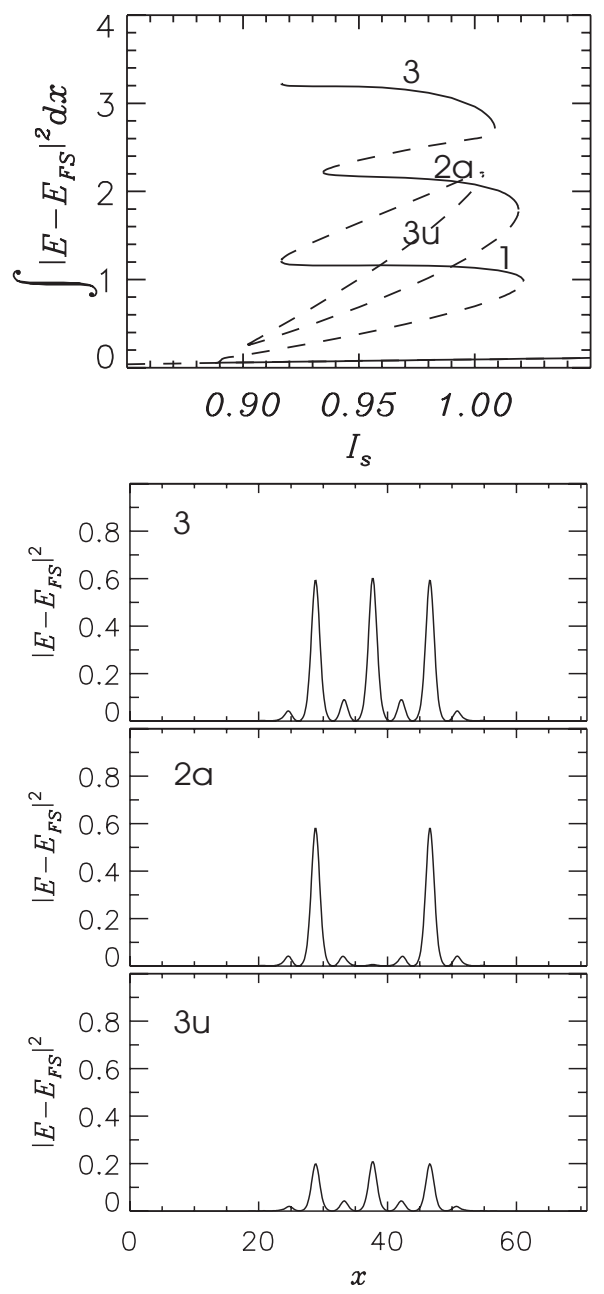

FIG. 9. Snaking of a single-peak localized structure. Solid (dashed) lines indicate stable (unstable) solutions. The lower panels show the transverse profile of the structures corresponding to lines labeled 3, 2a, and $3 \mathrm{u}$, respectively. The localized structure corresponding to line 1 is the single-peak CS shown in Fig. 8.

structures in a regime of bistability due to the ICPC. The existence of such localized structures is investigated in the next section.

Finally, on increasing the amplitude of the modulation even further, the unstable region shrinks in parameter space until it disappears completely. The fundamental solution is then the only possible stable output of the system. However, there is a resonance between the fundamental solution and the ICPC that modifies greatly the shape of the fundamental solution, as explained above.

In two transverse spatial dimensions the scenario is qualitatively the same for the first subcritical bifurcation. For larger values of the input pump, however, oscillatory instabilities hinder the behavior observed in one dimension (1D), and, in particular, prevent the observation of the additional subcritical region for large intracavity intensities. These oscillatory instabilities are commonplace in the 2D Kerr cavity model due to the collapse dynamics reminiscent of the solitons in the 2D nonlinear Schrödinger equation [9]. Figure 6 shows the coexisting 2D fundamental and pattern solutions 

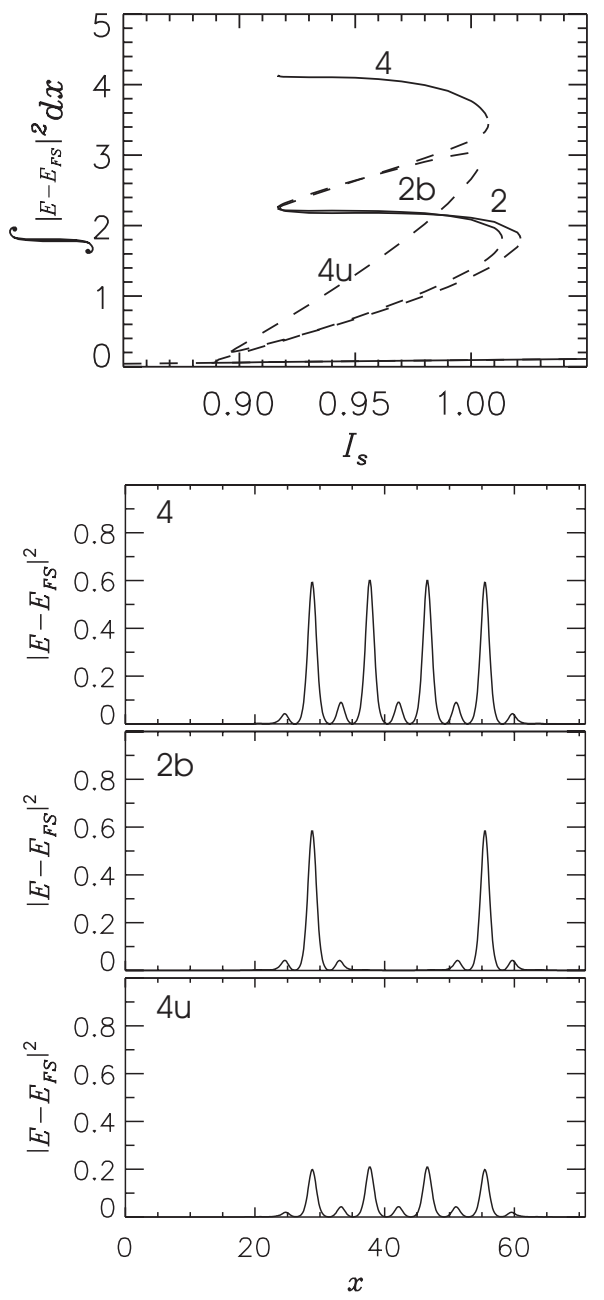

FIG. 10. Same as in Fig. 9 for the two peaks CS. In this case, line 2 corresponds to Fig. 12 below.

due to the modulational instability enhanced by the ICPC at lower values of $I_{s}$. Here we consider a photonic crystal with hexagonal symmetry and wave number $k_{p c}=\sqrt{3} k_{c}$.

\section{LOCALIZED STRUCTURES}

In this section we investigate the existence of CSs in the region of coexistence between the fundamental and the pattern solutions for appropriate values of the input pump. We consider here $\alpha=0.2$ and $\theta=1.5$ but the shape and character of the modulated CS remain unchanged for wide ranges of parameter values. Both solutions coexist for $0.891<I_{s}$ $<1.006$ [Fig. 5(b)]. Figure 7 shows the intensity profile of the fundamental and pattern solutions.

The fundamental solution is (trivially) modulated at the same periodicity $\lambda_{p c}=2 \pi / k_{p c}$ as the ICPC, while the pattern has a wavelength equal to $2 \lambda_{p c}$ and consists of high peaks on top of a lower modulation at $k_{p c}$. The coexistence of a fundamental and a pattern solution often leads to the existence of localized solutions (the CSs) that consist of an oscillation of the pattern embedded in the fundamental solution. Indeed, such solutions exist. Figure 8(a) shows the profile of a

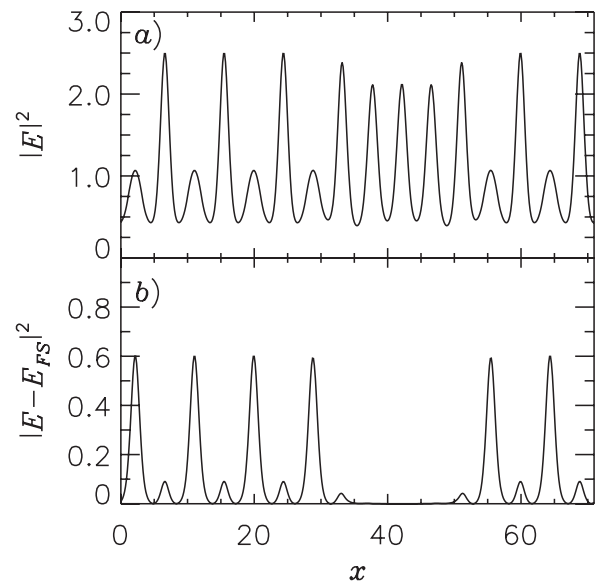

FIG. 11. Example of a complementary localized structure consisting of three oscillations of the fundamental solution surrounded by the pattern (a). This corresponds to two holes in the pattern (b). Again $I_{s}=0.96, \theta=1.5$, and $\alpha=0.2$.

single-peak CS generated by seeding a peak of the pattern on the fundamental solution. In order to recover more familiar shapes of CSs, we also plot the intensity of the difference between the CS and the fundamental solution $E_{F S}$ [see Fig. 8(b)].

This CS exists for $0.917<I_{s}<1.021$, a parameter range of size 0.104 . This range may appear small but it should be compared with its counterpart of 0.0087 for a CS without the ICPC. This region of CS existence is thus more than ten times broader than the original one. From an experimental point of view, this indicates that the CS might be far more easily created and observed in systems with an ICPC than without.

As for CSs in photonic systems with no background modulations, a family of (multipeaked) localized states whose bifurcation diagram presents snaking [10-12] is found. In Figs. 9 and 10 we show the snaking for the singleand two-peak CSs, respectively. We observe anomalous snaking with respect to the homogeneous case. In particular, in Fig. 9 we found that the single-peak CSs do not connect directly with the three-peak CSs as commonly observed in

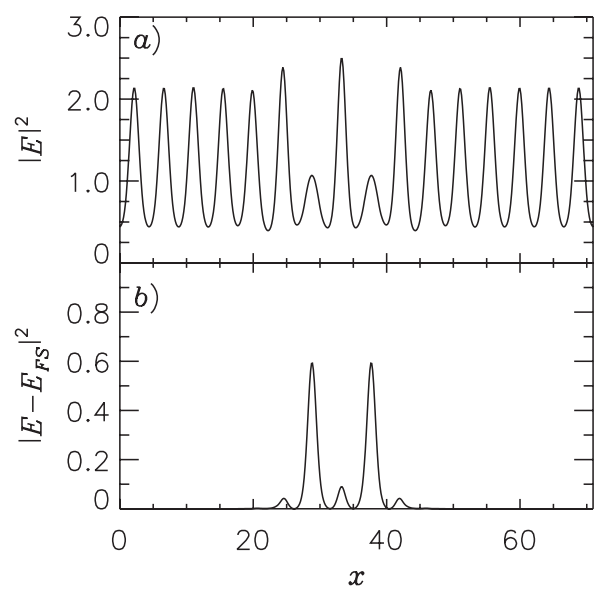

FIG. 12. Same as in Fig. 8 but for a two-peak CS. 


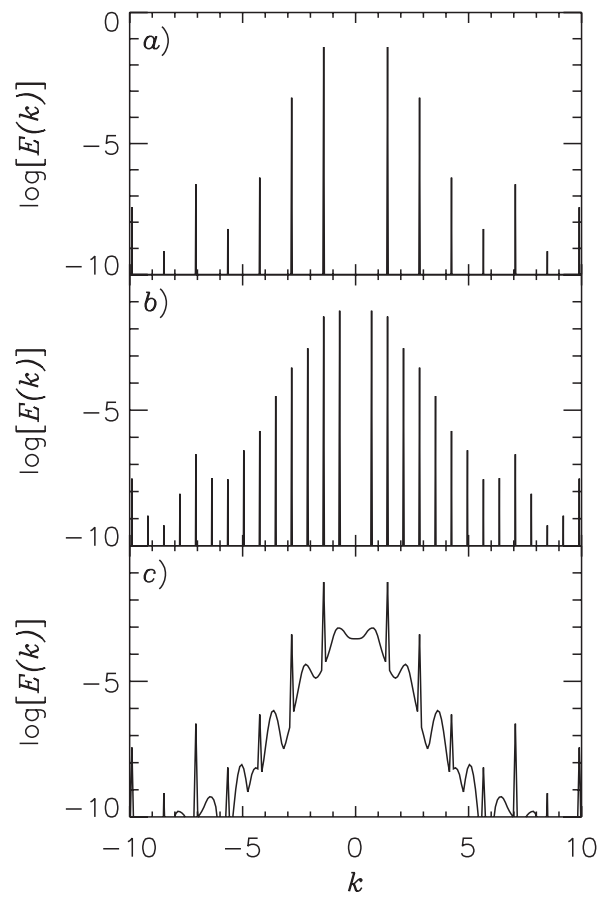

FIG. 13. Far field (Fourier transform) of (a) the fundamental, (b) the pattern, and (c) the single-peak CS. Again $I_{s}=0.96, \theta=1.5$, and $\alpha=0.2$.

homogeneous systems. In our case, the line of single-peak solutions (1) folds back first trough an unstable three-peak CS $(3 \mathrm{u})$, and then through a two-peak structure with a missing peak (hole) at its center (2a), i.e., a two-homoclinic orbit. In homogeneous systems $N$-homoclinic orbits appear in separated bifurcation lines, while in the ICPC system they merge with the fundamental snaking of one-homoclinic orbits. Similarly, in Fig. 10 the snaking of the fundamental two-peak CSs entangles with a two-homoclinic orbit consisting of two peaks with two holes in the middle (2b). The unusual properties and robustness of these structures cannot be explained by the general theory for normal CSs [10-12] since this should be modified to include the periodic forcing due to the ICPC. Localized states between two periodic solutions, although of a different nature from the ones investigated here, have also been studied in [13].

Together with CSs, one can also find the complementary localized structures consisting of holes in the pattern solution. Figure 11 shows an example of such a structure consisting of a two-hole localized structure in an extended pattern.

The CSs presented in Figs. 8, 11, and 12 may difficult to detect in the near-field intensity because of the limited excursion difference between the fundamental and pattern solutions. In of Figs. 8(b) and 12(b) we presented the intensity of the field difference between the CS and the fundamental solution. The localized character of the CS is now apparent. It is, however, difficult to perform difference measurements in real experiments since they require the storage of the fundamental solution and/or interferometric accuracy. An easier way to detect the presence of a CS in the modulated output of an ICPC device is to construct its far-field (Fourier trans-

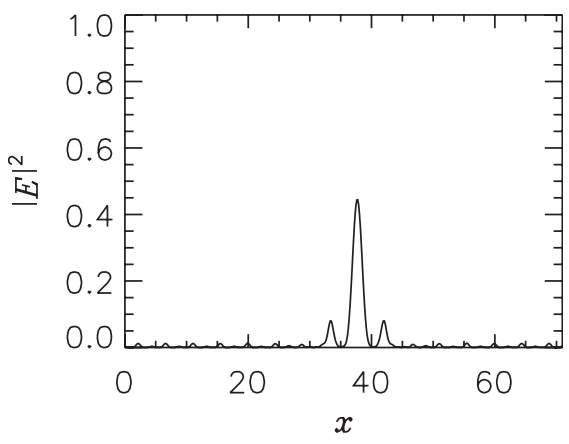

FIG. 14. Inverse Fourier transform of the far field of the CS of Fig. 8 after the wave-vector components of the fundamental solution have been filtered out. Again $I_{s}=0.96, \theta=1.5$, and $\alpha=0.2$.

form) distribution in the focal plane of a converging lens. Figure 13 shows the far field of the fundamental, the pattern, and the CS where the $k=0$ bias component has been removed. The broadening of the Fourier spectrum around $k$ $=0$ is a clear signature of the presence of the CS [see Fig. $13(\mathrm{c})]$ since it is due to the matching of the fundamental and pattern solutions that build the localized structure. We have observed similar broadenings for the entire family of CS solutions generated by the ICPC. In the far field it may be difficult, however, to distinguish between localized structures with different numbers of peaks. A solution is to use Fourier filtering in the far field to characterize the localization of the ICPC-induced CSs. In Fig. 14 we present the inverse Fourier transform of the CS after only the main Fourier component of the fundamental solution has been removed by appropriate filtering [2]. The result is basically the same as subtracting the whole fundamental solution [Fig. 8(b)]. Although the final signal has been attenuated, the localized character of the $\mathrm{CS}$ and its number of peaks are now evident.

Finally, for completeness, we present in Fig. 15 a localized solution in the presence of ICPC in two transverse dimensions. The parameter values are those corresponding to Fig. 6. We stress again that temporal oscillations prevent the observation of steady localized states in 2D in our model for large values of $I_{s}$ where the second subcritical regime is observed in 1D. However, for lower values of $I_{s}$ in the first

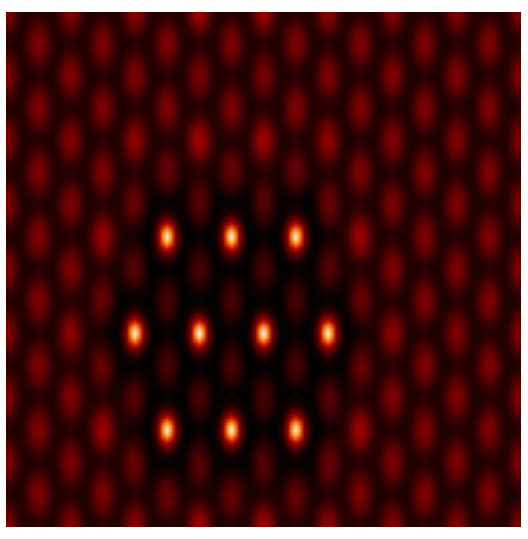

FIG. 15. (Color online) Same as in Fig. 6 for a ten peak localized solution. 
subcritical regime, stable localized peaks of the pattern are found embedded in the fundamental solution (see Fig. 15).

Elimination of the temporal oscillations in 2D may allow for the observation of steady localized structures around the second subcritical regime. This, together with the use of ICPC in other models of photonic devices to localize light in two transverse dimensions, is presently under investigation.

\section{CONCLUDING REMARKS}

We have studied the effects of a periodic modulation of the refractive index, i.e., a photonic crystal, on the formation of subcritical patterns and the existence of CSs. We have observed how the competition of linear effects of the ICPC such as the creation of a transverse photonic band gap, with nonlinearity leads to unexpected and useful phenomena. In subcritical regimes, the ICPC resonates with the cavity nonlinearity to progressively destroy patterned solutions. This is another aspect of the ICPC ability to inhibit modulational instabilities [3]. We have, however, demonstrated that for values of the modulation amplitude of the refractive index below the quenching of the instability new subcritical regions are created by the ICPC. These subcritical regimes appear as reverse bifurcations and are extremely wide (easily wider by a factor of 10) when compared to subcritical regions without the ICPC. Because of their large size, full families of localized states (CSs), due to the resonance between nonlinearity and photonic crystal, are nested in the bistability region of the subcritical bifurcations in both one and two transverse dimensions. These localized states represent a family of CSs connecting two spatially modulated solutions. Their detection in the near-field intensity distribution can be overshadowed by the lack of contrast between amplitudes of the two separate modulations. For this reason we have described two methods for CS detection. In the first one, we consider the intensity of the difference between the CS and the fundamental solution. This method works, but it may be difficult to implement experimentally since it requires the storage of the fundamental solution and/or interferometric accuracy. The second method for a clear detection of the CS induced by an ICPC is based on the far field and Fourier filtering, and can not only detect the localized nature of the CS but also reveal the number of peaks of the CS.

The results presented here have been obtained in a prototypical model for optical pattern formation, but their validity should extend to other systems more relevant for practical applications such as models for semiconductor lasers. Localized states, snaking, and subcritical bifurcations induced by periodic modulations of parameters are universal features in the study of complex systems and should be observable in a variety of scientific disciplines outside optics.

\section{ACKNOWLEDGMENTS}

D.G. acknowledges financial support from EPSRC (Grant No. GR/S28600/01), MEC (Grant No. TEC2006-10009), and Govern Balear (Grant No. PROGECIB-5A). G.L.O. thanks SGI, the CSDC, Roberto Livi of the University of Florence (Italy), and the EU Network FunFACS for financial support.
[1] R. Neubecker and A. Zimmermann, Phys. Rev. E 65, 035205(R) (2002).

[2] R. Martin, A. J. Scroggie, G.-L. Oppo, and W. J. Firth, Phys. Rev. Lett. 77, 4007 (1996); G. K. Harkness, R. Martin, A. J. Scroggie, G.-L. Oppo, and W. J. Firth, Phys. Rev. A 58, 2577 (1998).

[3] D. Gomila, R. Zambrini, and G.-L. Oppo, Phys. Rev. Lett. 92, 253904 (2004); D. Gomila and G.-L. Oppo, Phys. Rev. E 72, 016614 (2005).

[4] W. J. Firth and A. J. Scroggie, Phys. Rev. Lett. 76, 1623 (1996).

[5] S. Barland et al., Nature (London) 419, 699 (2002).

[6] L. A. Lugiato and R. Lefever, Phys. Rev. Lett. 58, 2209 (1987); W. J. Firth, A. J. Scroggie, G. S. McDonald, and L. A. Lugiato, Phys. Rev. A 46, R3609 (1992); L. A. Lugiato and C.
Oldano, ibid. 37, 3896 (1988).

[7] A. J. Scroggie et al., Chaos, Solitons Fractals 4, 1323 (1994).

[8] D. Gomila, A. J. Scroggie, and W. J. Firth, Physica D 227, 70 (2007).

[9] D. Gomila and P. Colet, Phys. Rev. A 68, 011801(R) (2003); W. J. Firth et al., J. Opt. Soc. Am. B 19, 747 (2002); D. Gomila, M. A. Matías, and P. Colet, Phys. Rev. Lett. 94, 063905 (2005).

[10] P. D. Woods and A. R. Champneys, Physica D 129, 147 (1999).

[11] P. Coullet, C. Riera, and C. Tresser, Phys. Rev. Lett. 84, 3069 (2000); Chaos 14, 193 (2004).

[12] J. Burke and E. Knobloch, Phys. Rev. E 73, 056211 (2006).

[13] U. Bortolozzo, M. G. Clerc, C. Falcon, S. Residori, and R. Rojas, Phys. Rev. Lett. 96, 214501 (2006). 